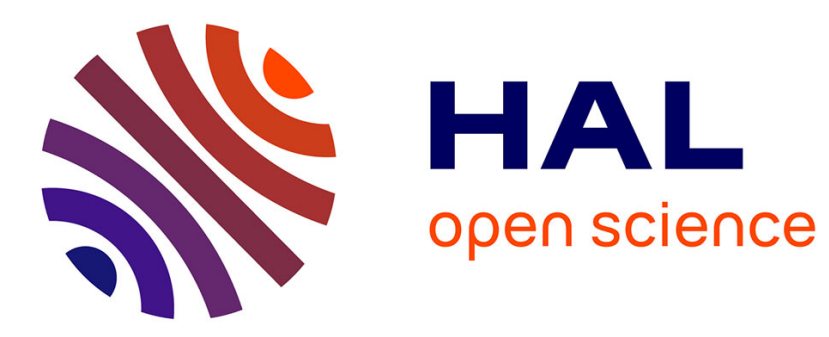

\title{
Nebulized antibiotics for ventilator-associated pneumonia: methodological framework for future multicenter randomized controlled trials
}

Antoine Monsel, Antoni Torres, Yinggang Zhu, Jerome Pugin, Jordi Rello, Jean-Jacques Rouby

\section{To cite this version:}

Antoine Monsel, Antoni Torres, Yinggang Zhu, Jerome Pugin, Jordi Rello, et al.. Nebulized antibiotics for ventilator-associated pneumonia: methodological framework for future multicenter randomized controlled trials. Current Opinion in Infectious Diseases, 2021, 34 (2), pp.156-168. 10.1097/QCO.0000000000000720 . hal-03190178

\section{HAL Id: hal-03190178 \\ https://hal.sorbonne-universite.fr/hal-03190178}

Submitted on 6 Apr 2021

HAL is a multi-disciplinary open access archive for the deposit and dissemination of scientific research documents, whether they are published or not. The documents may come from teaching and research institutions in France or abroad, or from public or private research centers.
L'archive ouverte pluridisciplinaire HAL, est destinée au dépôt et à la diffusion de documents scientifiques de niveau recherche, publiés ou non, émanant des établissements d'enseignement et de recherche français ou étrangers, des laboratoires publics ou privés. 


\title{
Nebulized antibiotics for ventilator-associated pneumonia: methodological framework for future multicenter randomized controlled trials
}

\author{
Antoine Monsel ${ }^{\mathrm{a}}$, Antoni Torres ${ }^{\mathrm{b}}$, Yinggang Zhu ${ }^{\mathrm{c}}$, Jerome Pugin ${ }^{\mathrm{d}}$, \\ Jordi Rello ${ }^{\mathrm{e}, \mathrm{f}, \mathrm{g}}$, Jean-Jacques Rouby ${ }^{\mathrm{a}}$, on behalf of the European Investigators \\ Network for Nebulized Antibiotics in Ventilator-associated Pneumonia \\ $(E N A V A P)^{*}$
}

\begin{abstract}
Purpose of review
Although experimental evidence supports the use of nebulized antibiotics in ventilator-associated pneumonia (VAP), two recent multicenter randomized controlled trials (RCTs) have failed to demonstrate any benefit in VAP caused by Gram-negative bacteria (GNB). This review examines the methodological requirements concerning future $\mathrm{RCTs}$.

\section{Recent findings}

High doses of nebulized antibiotics are required to reach the infected lung parenchyma. Breathsynchronized nebulizers do not allow delivery of high doses. Mesh nebulizers perform better than jet nebulizers. Epithelial lining fluid concentrations do not reflect interstitial lung concentrations in patients receiving nebulized antibiotics. Specific ventilator settings for optimizing lung deposition require sedation to avoid patient's asynchrony with the ventilator.

\section{Summary}

Future RCTs should compare a 3-5 day nebulization of amikacin or colistimethate sodium (CMS) to a 7-day intravenous administration of a new cephalosporine/B-lactamase inhibitor. Inclusion criteria should be a VAP or ventilator-associated tracheobronchitis caused by documented extensive-drug or pandrug resistant GNB. If the GNB remains susceptible to aminoglycosides, nebulized amikacin should be administered at a dose of $40 \mathrm{mg} / \mathrm{kg} /$ day. If resistant to aminoglycosides, nebulized CMS should be administered at a dose of 15 millions international units (IU)/day. In VAP caused by pandrug-resistant GNB, 15 millions IU/day nebulized CMS (substitution therapy) should be compared with a 9 millions IU/day intravenous CMS.

\section{Keywords}

breath-synchronized nebulization, epithelial lining fluid concentrations, nebulized amikacin, nebulized colistimethate sodium, nebulizers
\end{abstract}

\section{INTRODUCTION}

Animal experiments demonstrate that nebulized aminoglycosides and polymyxins produce high lung tissue concentrations and provide rapid and efficient bacterial killing in the lung parenchyma infected by Gram-negative bacteria (GNB) [1]. These

\footnotetext{
${ }^{a}$ Multidisciplinary Intensive Care Unit, Department of Anaesthesiology and Critical Care, Sorbonne University of Paris, La Pitié-Salpêtrière Hospital, Assistance Publique Hôpitaux de Paris, Paris, France, 'D Department of Pulmonology, Hospital Clinic of Barcelona, University of Barcelona, Institut d'Investigacions Biomèdiques August Pi i Sunyer (IDIBAPS), SGR 911-Ciber de Enfermedades Respiratorias (CIBERES), Barcelona, Spain, ${ }^{\circ}$ Department of Pulmonary and Critical Care Medicine, Fudan University, Hua-dong Hospital, Shanghai, China, Intensive Care Division, University of Geneva, University Hospitals of Geneva, Geneva, Switzerland, ${ }^{\circ}$ Centro de Investigación Biomédica en Red (CIBERES), Instituto de Salud Carlos III, Madrid, ${ }^{\mathrm{f}} \mathrm{Clinical}$ Research \& Innovation in Pneumonia \& Sepsis, Vall d'Hebron Institute of Research (VHIR), Barcelona, Spain and ${ }^{\mathrm{g}} \mathrm{Clinical}$ Research, CHU Nîmes, Université Montpellier-Nîmes, Nîmes, France

Correspondence to Pr Jean-Jacques Rouby, Sorbonne University, Paris, France. Tel: +33 6298354 20; e-mail: jjrouby@invivo.edu *The names of the members of the European Investigators Network for Nebulized Antibiotics in Ventilator-associated Pneumonia (ENAVAP) are listed at the end of the article.
}

Curr Opin Infect Dis 2021, 34:156-168

DOI:10.1097/OCO.0000000000000720 


\section{KEY POINTS}

- To optimize lung deposition of nebulized antibiotic in VAP, specific ventilator settings should be used during nebulization to reduce inspiratory flow velocity; humidification and warming of inspired gas should be interrupted to avoid a rainout effect in the circuits and airways, and sedation administered to avoid dyssynchrony with the ventilator.

- In ventilated patients treated by nebulized antibiotics, epithelial lining fluid antibiotic concentrations markedly overestimate lung interstitial space fluid concentrations due to the bronchial contamination of the fluid lavage.

- Future multicentre RCTs on nebulized amikacin and colistimethate sodium should include patients with VAP and ventilator-associated tracheobronchitis caused by extensive-drug and pandrug resistant gram-negative bacteria.

- Nebulization of amikacin or colistimethate sodium alone should be compared with a 7-day intravenous administration of new cephalosporine/B-lactamase inhibitors (monotherapy) or colistimethate sodium.

- High doses amikacin $(40 \mathrm{mg} / \mathrm{kg} /$ day) and colistimethate sodium (15 millions IU/day) should be administered using continuous rather inspirationsynchronized nebulization for 3-5 days.

- Expected benefits from nebulized antibiotics are a shorter time to clinical cure, a decrease in colistimethate sodium-induced renal toxicity, a shorter duration of antibiotic administration and a reduction of mechanical ventilation duration.

benefits were demonstrated using postmortem lung tissue homogenates [2,3] and in-vivo microdialysis [4"']. Despite experimental evidence of efficacy, clinical studies in ventilator-associated pneumonia (VAP) have failed to provide convincing results. Multicentre randomized controlled trials (RCTs) are sparse, have used nonoptimized techniques of nebulization, have enrolled heterogeneous populations of patients with hospital-acquired pneumonia or VAP and failed to demonstrate superiority for the nebulization route $\left[5,6^{\mathbf{*}}, 7\right]$. As a consequence, American and European academic societies do not recommend the routine use of nebulized antibiotics to treat VAP [8-12]. In 2016, American guidelines suggested that VAP due to GNB that are susceptible to only aminoglycosides or polymyxins could be treated with both nebulized and intravenous agents (adjunctive therapy) [8]. In 2017, the French guidelines suggested the use of nebulized colistimethate sodium (CMS) and/or aminoglycosides in VAP caused by GNB sensitive only to CMS and/or aminoglycosides [11]. In this opinion article, reflecting the view of the European Investigators Network for Nebulized Antibiotics in Ventilator-associated Pneumonia [13"], we analyse potential underlying reasons that led multicenter RCTs to fail, and we propose various insights to improve methods of future RCTs on nebulized amikacin and CMS in patients with VAP caused by GNB.

\section{PATIENTS WITH VENTILATOR- ASSOCIATED PNEUMONIA WHO MAY BENEFIT FROM NEBULIZED AMIKACIN AND COLISTIMETHATE SODIUM}

In theory, VAP caused by microorganisms susceptible only to antibiotics with limited pulmonary penetration, could benefit from the nebulization route. Lung tissue penetration is excellent for quinolones, variable for ß-lactams and weak for glycopeptides, aminoglycosides and polymyxins [14]. As a consequence, aminoglycosides and CMS are the main antibiotics whose nebulization could provide clinical benefit. Recently experimental data suggest that such benefit could also be obtained by nebulized vancomycin [15"].

Treatment of VAP by mono or dual combination therapy is a key methodological issue for future RCTs. Adding intravenous aminoglycosides or fluoroquinolones to $\beta$-lactams is not more efficient than intravenous $\beta$-lactams alone to treat sepsis and VAP $[16,17]$. For the initial empirical therapy, European and American guidelines recommend an intravenous bitherapy in patients at risk of VAP caused by multidrug resistant GNB $[8,10,11]$. American guidelines suggested to continue bitherapy in patients with septic shock [18] and documented multidrug resistant GNB [8]. European guidelines suggested to continue bitherapy only in patients with documented extensive-drug resistant (XDR) GNB [10]. French recommendations suggested to use monotherapy after the identification of the causative microorganism, considering the lack of evidence supporting the benefit of bitherapy [11].

Refering to the stratification of GNB resistance (Fig. 1) [19], patients with VAP caused by XDR GNB susceptible to aminoglycosides, could benefit from nebulized amikacin whereas patients with VAP caused by XDR GNB resistant to aminoglycosides could benefit from nebulized CMS. Compared with the intravenous administration of a new cephalosporine/ß-lactamase inhibitor, the nebulization of amikacin and CMS may have several advantages: reduction of bacterial resistance in the ICU [20], more efficient treatment of ventilator-associated tracheobronchitis (VAT) due to the very high tracheobronchial antibiotic concentrations [21], and shorter duration of treatment allowing to reduce antibiotic pressure in the ICU. 


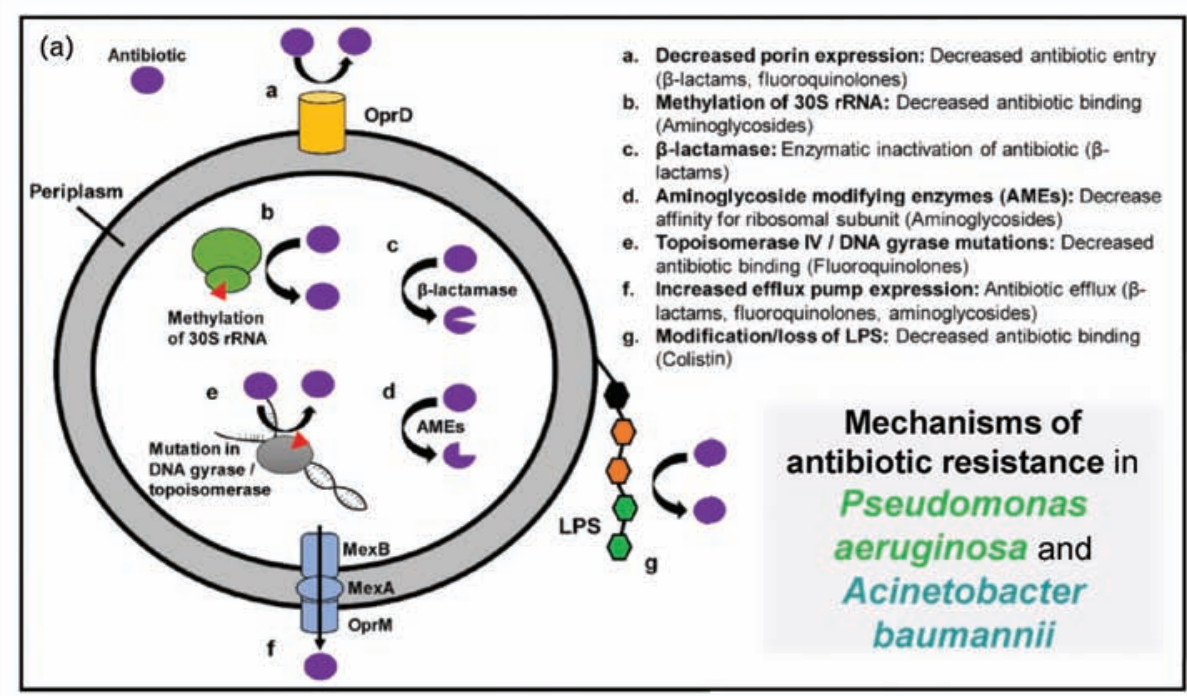

\begin{tabular}{|c|c|c|c|}
\hline (b) & Antibiotic & $\begin{array}{l}\text { Pseudomonas } \\
\text { aeruginosa with } \\
\text { extensive-drug } \\
\text { resistance }\end{array}$ & $\begin{array}{l}\text { Pseudomonas } \\
\text { aeruginosa with } \\
\text { multidrug } \\
\text { resistance }\end{array}$ \\
\hline \multirow{3}{*}{ Aminoglycosides } & Gentamycin & $x$ & $\mathrm{X}$ \\
\hline & Tobramycin & $\mathrm{X}$ & $\mathrm{X}$ \\
\hline & Amikacin & $\mathrm{X}$ & \\
\hline \multirow{3}{*}{$\begin{array}{l}\text { Antipseudomonal } \\
\text { carbapenems }\end{array}$} & Imipenem & $\mathrm{X}$ & $\mathrm{X}$ \\
\hline & Meropenem & $\mathrm{X}$ & $\mathrm{X}$ \\
\hline & Doripenem & $\mathrm{X}$ & $\mathrm{X}$ \\
\hline \multirow{3}{*}{$\begin{array}{l}\text { Antipseudomonal } \\
\text { cephalosporins }\end{array}$} & Ceftazidime & $\bar{X}$ & $\mathrm{X}$ \\
\hline & Cefepime & $\mathrm{X}$ & $\mathrm{X}$ \\
\hline & Cefiderocol & & \\
\hline \multirow{2}{*}{$\underset{\beta \text {-lactamases inhibitors }}{+}$} & $\begin{array}{l}\text { Ceftazidime } \\
\text { avibactam }\end{array}$ & & \\
\hline & $\begin{array}{l}\text { Ceftozolane } \\
\text { tazobactam }\end{array}$ & & \\
\hline \multirow{2}{*}{$\begin{array}{l}\text { Antipseudomonal } \\
\text { fluoroquinolones }\end{array}$} & Ciprofloxacin & $\bar{X}$ & $\mathrm{X}$ \\
\hline & Levofloxacin & $X$ & $\mathrm{X}$ \\
\hline \multirow{2}{*}{$\begin{array}{l}\text { Antipseudomonal penicillins } \\
+\beta \text {-lactamases inhibitors }\end{array}$} & $\begin{array}{l}\text { Piperacillin } \\
\text { tazobactam }\end{array}$ & $\mathbf{X}$ & $\mathbf{X}$ \\
\hline & $\begin{array}{c}\text { Ticarcillin } \\
\text { clavulanic acid }\end{array}$ & $\mathbf{X}$ & $\mathrm{X}$ \\
\hline Phosphonic acids & Fosfomycin & $\mathrm{X}$ & \\
\hline Polymyxins & Colimycin & & \\
\hline
\end{tabular}

FIGURE 1. Resistance of Gram-negative bacteria causing ventilator-associated pneumonia. (a) Mechanisms of resistance for Pseudomonas aeruginosa and Acinetobacter baumannii; (b) antibiotic sensitivity of two specimens of Pseudomonas aeruginosa with multidrug resistance and extensive - drug resistance. (a) - Reproduced with permission from Eichenberger EM, Thaden JT. Epidemiology and mechanisms of resistance of extensively drug resistant Gram-negative bacteria. Antibiotics 2019;8:pii: 37. 
The limited penetration of nebulized antibiotics into consolidated pneumonic lung and the possible long delay before they reach the sites of infection, could justify the concomitant administration of nebulized and intravenous antibiotics in VAP where 'lobar' consolidation is often present. Experimentally, the alveolar deposition of nebulized antibiotics decreases with obstruction of distal bronchioles by purulent plugs, the histologic severity of pneumonia and the loss of lung aeration [1]. Severity of lung aeration loss has opposite effects depending on whether antibiotics are intravenously administered or nebulized: tissue concentrations increase in case of intravenous administration and decrease after nebulization $[2,3,22]$. Very likely, the increased permeability of the alveolar-capillary barrier in VAP, promotes intravenous amikacin penetration into the lung, whereas the multiple purulent plugs in distal bronchioles limit its alveolar deposition. However, within consolidated pneumonic lung regions, amikacin and colistin nebulized lung tissue concentrations remain largely above minimal inhibitory concentrations $[3,22]$, raising the question how nebulized antibiotics reach infected lung parenchyma despite the lack of any pulmonary aeration. Intraparenchymal pseudocysts and bronchiolar distension frequently observed in consolidated lung areas of ventilated animals (and patients) with VAP [23], likely represent one of the routes by which nebulized antibiotics reach infected lung regions devoid of alveolar aeration. In addition, there is a continuum between the bronchial and the interstitial compartment. Any consolidation is contiguous with the bronchi which opens the door for nebulized antibiotics. According to these experimental data, it seems reasonable to foster nebulization alone.

Bacteremic VAP caused by XDR GNB is a clinical situation where nebulization and intravenous administration should be combined. Systemic diffusion of nebulized aminoglycosides and CMS do not achieve systemic bactericidal concentrations.

\section{DETERMINATION OF THE OPTIMAL NEBULIZED DOSE}

The understanding of mechanisms determining lung parenchymal deposition and the use of appropriate doses are key issues for therapeutic efficiency.

\section{Extrapulmonary, bronchial and lung parenchymal deposition}

In spontaneously breathing patients with cystic fibrosis, around $70 \%$ of nebulized antibiotics reach the infected bronchial tree. In ventilated patients with VAP, less than $40 \%$ reach the infected lung parenchyma (Fig. 2) [24].

Massive inertial impaction of the aerosol in the ventilator circuits and tracheo-bronchial walls explains the limited pulmonary deposition. The retention in the nebulizer varies from 50\%, with jet nebulizers to less than $5 \%$ with mesh nebulizers [24]. Heat and moisture exchangers significantly reduce the inhaled mass by trapping aerosolized particles in the filter [25]. Warming and humidifying inspired gas, increases aerosol particle size and induces a rainout effect in respiratory circuits and conducting airways. Non humidified aerosol increases inhaled mass delivery and lung deposition. In VAP, dry nebulization increased gentamicin, amikacin or vancomycin sputum levels by 3.63 compared with humidified nebulization [26]. With dry nebulization and optimized ventilator settings, circuit and bronchial deposition reach an average of 30 and $35 \%$, respectively [24].

\section{Bronchial contamination of the epithelial lining fluid}

The bronchial deposition of nebulized antibiotics skews the epithelial lining fluid (ELF) concentrations [27"]. In animals receiving a single aerosol dose of CMS or tobramycin, the ELF antibiotic concentrations measured $1 \mathrm{~h}$ after nebulization ranged between 700 and $4000 \mu \mathrm{g} / \mathrm{ml}\left[4^{*}, 21,28,29\right]$. In healthy ewes receiving a 400-mg aerosol dose of tobramycin, the ELF concentrations were 100-fold higher than the interstitial space fluid concentrations measured using microdialysis [4"'], clearly suggesting a massive bronchial contamination during the bronchoalveolar lavage procedure [27"]. In critically ill patients with VAP treated by nebulized CMS [30] or amikacin [31], bronchial concentrations above $1000 \mu \mathrm{g} / \mathrm{ml}$ were found.

To get samples representative of ELF, the fiberscope is advanced as far as possible in the bronchial tree. Then, three to five aliquots of $20-60 \mathrm{ml}$ saline are instilled and the aspirate from the third aliquot is used for ELF analysis. As shown in Fig. 3a, a $60 \mathrm{ml}$ aliquot lavages a large segmental area [32]. Following nebulization, the tracheobronchial tree is coated with aerosolized antibiotic. The fiberscope is contaminated during its passage and the ELF antibiotic concentrations reflect more bronchial than interstitial lung tissue concentrations. In sheep receiving $10 \mathrm{mg} / \mathrm{kg}$ nebulized amikacin, tracheobronchial concentrations were measured more than $10,000 \mu \mathrm{g} / \mathrm{ml}$ at the end of nebulization (Fig. 3b) [21]. In critically ill patients with VAP receiving $5 \mathrm{mg} / \mathrm{kg}$ of nebulized amikacin, tracheobronchial and ELF amikacin concentrations ranged between 


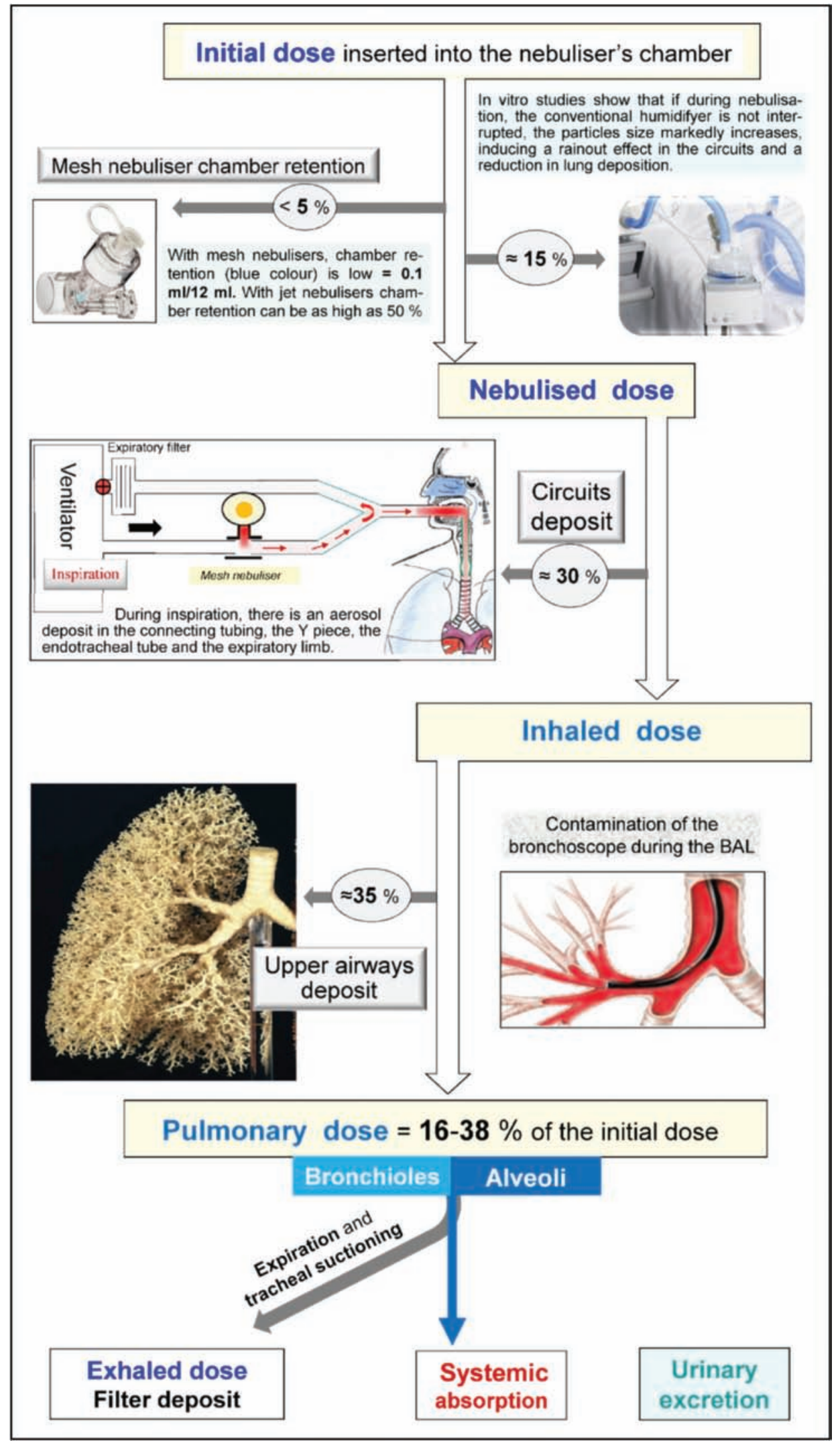

FIGURE 2. Extrapulmonary and pulmonary deposition of nebulized antibiotics. Reproduced with permission [50]. Copyright ${ }^{\circ}$ 2020 Elsevier Masson SAS. All rights reserved. 


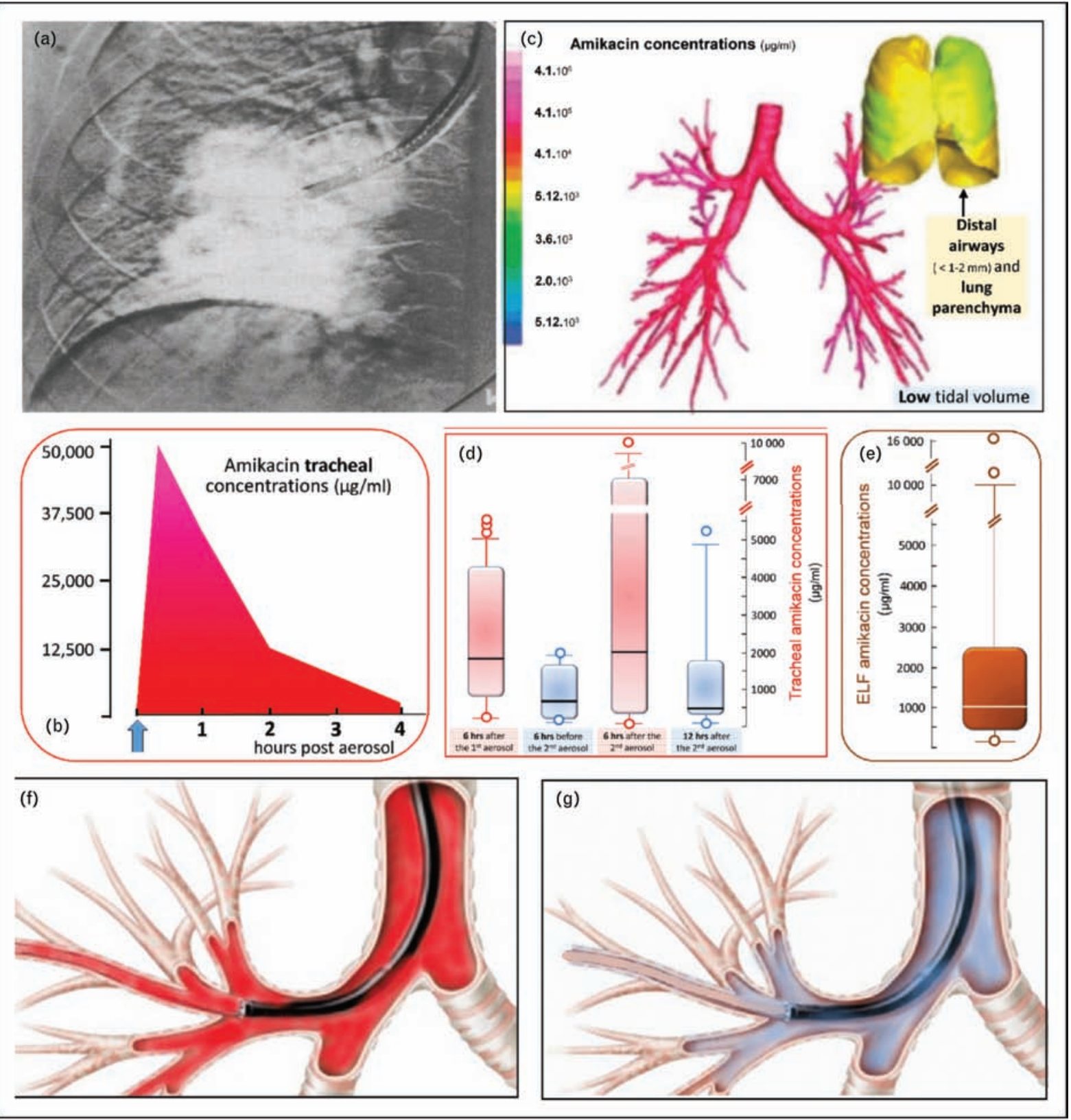

FIGURE 3. Bronchial contamination of the epithelial lining fluid during the fiberoptic bronchoalveolar lavage procedure. (a) Distribution of bronchoalveolar lavage fluid as assessed by digital subtraction radiography. The aspirate from the third 60-ml aliquot is issued from the entire volume of the medial segment of the middle lobe; (b) amikacin tracheal concentrations measured before and $10 \mathrm{~min}, 2 \mathrm{~h}$ and $4 \mathrm{~h}$ after a single nebulization of amikacin $300 \mathrm{mg}$ in five pigs with Pseudomonas aeruginosa inoculation pneumonia; (c) distribution of tobramycin concentrations between proximal and lung parenchyma immediately after the nebulization of $600 \mathrm{mg}$ in patients with cystic fibrosis (colour scale indicates amikacin concentrations ranging from 4,100,00 $\mathrm{\mu g} / \mathrm{ml}$ to $512 \mu \mathrm{g} / \mathrm{mll}$. Aerosol concentrations were computed using airway models reconstructed from computed tomography scans of patients with cystic fibrosis, in combination with computational fluid dynamic simulations. Proximal airways defined as bronchi with an internal diameter greater than $1 \mathrm{~mm}$ are represented as the tracheal bronchial tree, whereas distal airways are represented as lung parenchyma. Nebulization was simulated using a PARI-LCR Plus nebulizer (Midlothian, Texas, USA); (d) amikacin tracheal concentrations measured in 19 patients with ventilator-associated pneumonia treated by two daily aerosol doses of $400 \mathrm{mg}$, in the $6 \mathrm{~h}$ after the first and second aerosol of day 3 (light red boxplots), and in the $6 \mathrm{~h}$ preceding the second aerosol of day 3 and the first aerosol of day 4 (light blue boxplots); (e) epithelial lining fluid amikacin concentrations measured on day 3 of treatment in 28 patients $(f)$ and $(g)$ contamination of the fibroscope by high bronchial concentrations (red colour corresponding to the colour scale diplayed in c) immediately after the end of the nebulization and by low bronchial concentrations (blue colour corresponding to the colour scale diplayed in c) immediately before the next nebulization. Reproduced with permission [27"]. 
500 and $16,000 \mu \mathrm{g} / \mathrm{ml}$ (Fig. 3d and e) [31]. Although simulation indicates a concentration gradient between proximal airways and lung parenchyma (Fig. 3c), tracheobronchial and ELF amikacin concentrations were increased in the same proportions (Fig. 3d and e), reinforcing the hypothesis of massive bronchial contamination. To quantify lung tissue concentrations and the 'bronchial contamination' over time, future experiments should compare bronchial, ELF using bronchoalveolar lavage and lung interstitial concentrations using microdialysis [4"-] after the single nebulization of a high dose of nebulized aminoglycoside or CMS to large animals with inoculation pneumonia.

In conclusion, ELF antibiotic concentrations in patients receiving nebulized antibiotics markedly overestimate lung interstitial concentrations and are not appropriate for assessing lung tissue concentrations. As microdialysis catheters cannot be inserted routinely in patients, microneedles and aptamerbased probes could be an attractive option [33].

\section{Toxicity risk and rationale to determine the nebulized dose}

Local and general toxicity of nebulized aminoglycosides and CMS depend on the nebulized dose and the systemic diffusion from the respiratory system. Whereas systemic diffusion of nebulized aminoglycosides is limited in healthy lung, it markedly increases when the alveolar-capillary barrier is disrupted by the inflammatory process [13"]. In animals with inoculation pneumonia, trough amikacin concentrations are not different between nebulization and intravenous administration when the nebulized dose is equal to the intravenous dose augmented by extrapulmonary deposition $\left[1,13^{-}\right]$. As a consequence, the risk of amikacin toxicity is not reduced with nebulization. A possible rationale for dosing is to provide equivalent doses to the respiratory system either by the endotracheal tube (nebulization) or by the pulmonary artery (intravenous administration). In other words: nebulization dose $=$ intravenous dose + extrapulmonary deposition dose (Fig. 4). High doses should therefore be administered, amounting $40 \mathrm{mg} / \mathrm{kg} /$ day. As aminoglycosides are concentration dependant antibiotics with a postantibiotic effect, a single nebulization per day may be prefered [13"].

In contrast, systemic diffusion of nebulized CMS is low, even in presence of extensive inoculation pneumonia [3] or VAP [34]. As a consequence, the rationale for dosing is different, mainly determined by the bronchial tolerance of the aerosol. As nebulized CMS has a good tracheobronchial tolerability, doses can be increased far above the intravenous dose augmented by extrapulmonary deposition. To increase the bactericidal activity (CMS is a concentration dependant antibiotic), up to 5 million international units (IU) can be nebulized each $8 \mathrm{~h}$ and compared with 3 million IU intravenously administered each $8 \mathrm{~h}$.

\section{Substitution rather than adjunctive therapy}

The rationale for adjunctive therapy (nebulization and intravenous administration) appears limited [1]. For aminoglycosides, adjunctive therapy increases lung tissue and trough plasma concentrations. Therefore, the risk of toxicity likely increases. For CMS, adjunctive therapy increases lung tissue but not plasma concentrations. Therefore, it likely improves efficacy with a similar risk of toxicity. In contrast, substitution therapy markedly reduces colistin plasma concentrations and decreases the risk of toxicity as shown in a recent meta-analysis [35]. Therefore, the European Society of Clinical Microbiology and Infectious Diseases recommended to compare substitution therapy rather than adjunctive therapy with intravenous administration in future RCTs [36].

\section{TECHNIQUE OF NEBULIZATION}

To optimize lung deposition in ventilated patients, a continuous rather than breath-actuated nebulization should be provided using mesh nebulizers together with specific ventilator settings to reduce flow velocity and inertial impaction of aerosolized particles.

\section{Continuous rather than inspiration- synchronized nebulization}

Breath-synchronized mesh nebulizers are softwaredriven devices that monitor inspiratory flow, respiratory rate and inspiratory time. They increase the respirable mass $[26,37]$ but markedly lengthen the time of nebulization by restricting aerosol generation to the inspiratory phase [38"]. With nebulizers designed to synchronize aerosol generation to the first $75 \%$ of the inspiratory phase, the time of nebulization per minute is independent of the respiratory frequency and can be calculated as follows:

$$
\begin{aligned}
& \text { Nebulization time }(\mathrm{s} / \mathrm{min}) \\
& =60 \quad 0.75 \quad \mathrm{Ti} / \text { Ttot }
\end{aligned}
$$

Compared with a continuous mesh nebulizer, an inspiration-synchronized mesh nebulizer extends the time of nebulization by three to nine fold. Nebulization of amikacin $25 \mathrm{mg} / \mathrm{kg}$ takes 30 


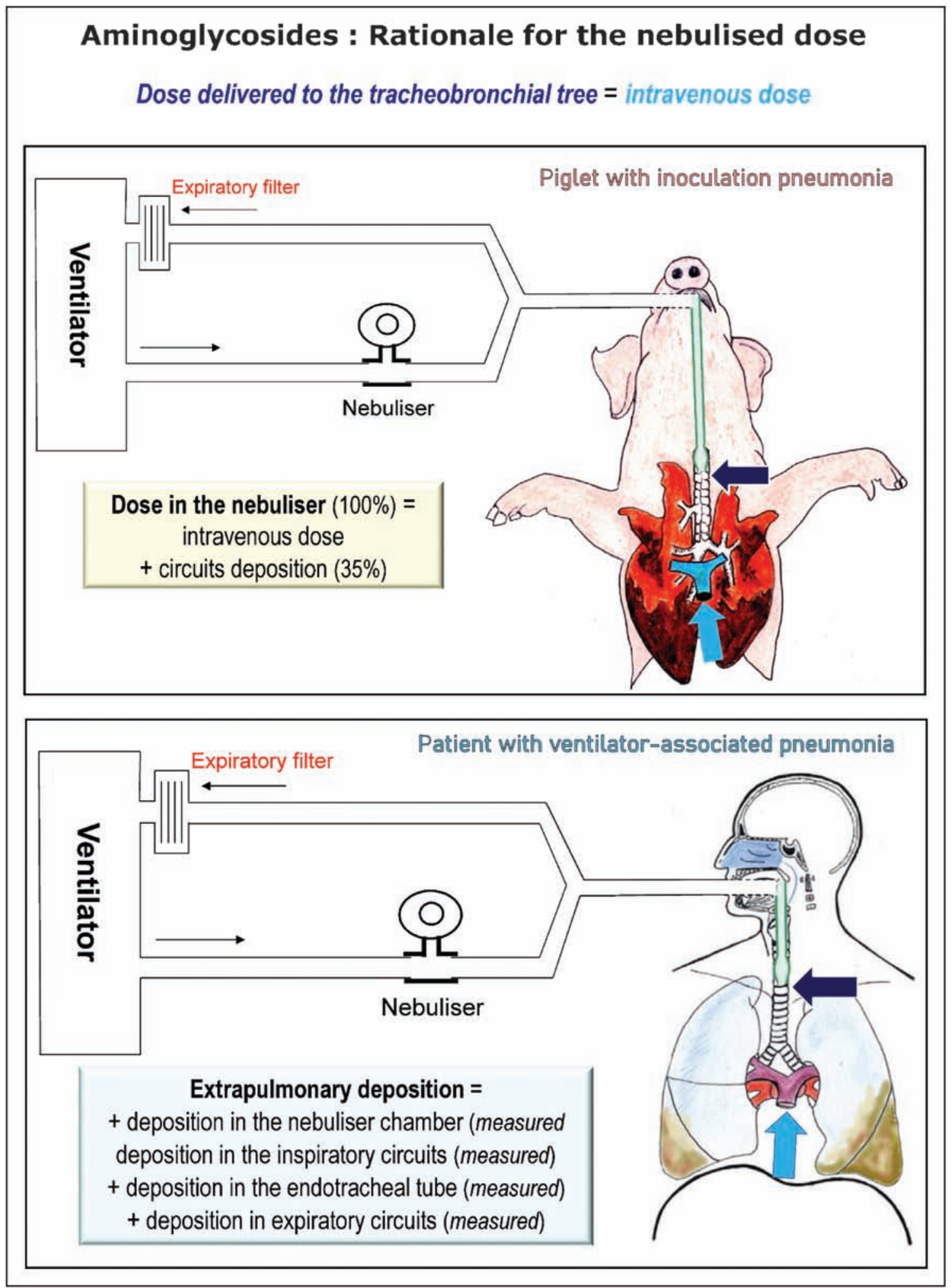

FIGURE 4. Rationale for determining equivalent dosing between nebulization and intravenous administration of aminoglycosides. Ventilated piglets with inoculation pneumonia and patients with ventilator associated pneumonia receive the same dose of aminoglycoside at the distal tip of the endotracheal tube (dark blue arrow, nebulization) and in the pulmonary artery (light blue arrow, intravenous administration). The dose inserted into the chamber of the nebulizer is equal to the intravenous dose and extrapulmonary deposition. Extrapulmonary deposition can be measured by washing out inspiratory and expiratory circuits and the endotracheal tube by a know volume of saline and measuring the mean concentration of amikacin in the collected liquid [39]. Reproduced and modified with permission [1]. 
45 min using continuous mesh nebulizers [39], whereas nebulization of $12 \mathrm{mg} / \mathrm{kg}$ takes $60 \mathrm{~min}$ using inspiration-synchronized mesh nebulizers [31]. As a consequence, nebulization of amikacin $40 \mathrm{mg} / \mathrm{kg} /$ day, the dose recommended in XDR GNB $\mathrm{VAP}$, is unfeasible using inspiration-synchronized mesh nebulizers as it would require two $3-5 \mathrm{~h}$ nebulizations per day [38"].

Continuous mesh nebulizers generate the antibiotic aerosol throughout the respiratory cycle. During expiration, the aerosol accumulates in the inspiratory limb and is propelled into the airways during the next inspiration (bolus effect). The 'bolus effect' contributes to the delivery of highly concentrated aerosol to the tracheobronchial tree and the distal lung and justifies the use of continuous mesh nebulizers in VAP.

\section{Optimization of ventilator settings during the nebulization phase}

Limiting inspiratory flow velocity reduces inertial impaction of the aerosol in the airways and optimizes lung deposition. To achieve that goal, it is recommended to use specifically designed smooth angles and inner surface tubings $[36,39]$ and to select specific ventilator settings during the nebulization [36]: volume-controlled ventilation with a constant inspiratory flow, Inspiratory/Expiratory ratio at $1: 1$, respiratory rate at $12-15 \mathrm{bpm}$, tidal volume at $8 \mathrm{ml} / \mathrm{kg}$, and absence or minimum bias flow (21/min). In patients with acute respiratory distress syndrome, such ventilator settings generate asynchronies which should be controlled by increasing sedation level transitorily. A plateau end-inspiratory pause of $20 \%$ should be set to promote aerosol deposition on alveolar walls. Pressure support should be discouraged because the decelerating inspiratory flow produces turbulences [40]. The heat and moisture exchanger should be removed and heated humidifiers stopped to avoid hygroscopic growth of the aerosolized particles and a rainout effect in the endotracheal tube and airways. To avoid aerosol waste in the expiratory limb, the nebulizer should be positioned $15 \mathrm{~cm}$ before the Y piece, on the inspiratory limb $[36,39]$.

\section{Advantages of mesh nebulizers over jet nebulizers}

In-vitro and in-vivo studies suggest the superiority of mesh over jet nebulizers for nebulizing antibiotics and bronchodilators [41-45]. With both types, mass median aerodynamic diameters remain below $5 \mu \mathrm{m}$ [41-43], the threshold above which the majority of aerosolized particles cannot reach the deep lung. Fluorescence lung imaging [42] and scintigraphic studies $[40,44]$, demonstrate that mesh nebulizers provide a higher respirable inhaled mass than jet nebulizers. Another advantage is a much smaller residual volume $(46 \%$ with jet nebulizers versus $4 \%$ with mesh nebulizers) that allows the administration of high doses in a shorter duration of time. In addition there is no interference with inhaled gas delivered by the ventilator. In spontaneously breathing anaesthetised mice inhaling salbutamol in an exposure chamber, postmortem tissue and serum concentrations were $60 \%$ higher with mesh compared with jet nebulizers [42], whereas residual volume was significantly reduced. In moderate to severe chronic obstructive pulmonary disease patients on noninvasive ventilation, mesh nebulizers deposited more than three-fold more radioaerosol into the lungs than jet nebulizers [44]. The technical advantages of mesh nebulizers may affect outcome. In a series of 1594 patients with asthma and chronic obstructive pulmonary disease admitted to a US Emergency Department and treated by bronchodilators, the use of mesh nebulizers $(n=715)$ was associated with fewer admissions to the hospital, shorter length of stay in the emergency department and a reduction in albuterol dose [45].

A note of caution, however, should be added concerning the viability of vibrating mesh technology. An unexpected $30 \%$ rate of premature cessation of aerosol production has been reported with the Aerogen mesh nebulizer [46]. Failure was associated with a wide range of residual volume averaging $21.3 \%$ of the charge compared with $1.5 \%$ of the charge when the nebulization was successful. In contrast, older jet nebulizers have been shown to be reliable with repeated use [47].

\section{FUTURE RANDOMIZED CONTROLLED TRIALS}

The reasons why the IASIS [5] and INHALE [6"] multicenter RCTs failed, have been extensively analysed $\left[38^{*}, 48-50\right]$ : first, inclusion of patients with VAP caused by susceptible GNB; second, adjunctive therapy combining two even three antibiotics to treat VAP without shock and/or not caused by multiresistant GNB; third, use of inspiration-synchronized mesh nebulizers imposing the administration of low-dose amikacin; fourth, lack of optimization of mechanical ventilation during the nebulization phase; fifth, low-dose of nebulized amikacin based on high ELF concentrations reflecting rather bronchial than lung interstitial concentrations.

Future RCTs should compare the nebulization of amikacin and CMS with the parenteral administration of new cephalosporines/ß-lactamase inhibitors in patients with VAP or VAT caused by XDR GNB. Ceftazidime avibactam and ceftolozane tazobactam 
are active against Enterobacterales, Pseudomonas aeruginosa and Klebsiella pneumoniae producing carbapenemase and oxacillinase (a main challenge in Mediterranean countries), and cefiderocol or eravacycline are active against carbapenem-resistant Acinetobacter baumannii. If the GNB remains susceptible to aminoglycosides, nebulized amikacin should be compared with the intravenous cephalosporine/ßlactamase inhibitor. If the GNB is resistant to aminoglycosides, nebulized CMS should be compared with the intravenous cephalosporine/ß-lactamase inhibitor. In VAP caused by documented pandrug resistant GNB, nebulized CMS should be compared with intravenous CMS (subsitution therapy). Benefits that can be expected are a more rapid clinical cure, a reduction of duration of mechanical ventilation, a reduction of renal toxicity in case of subsitution therapy using CMS, and a shorter duration of antibiotic administration. As lung deposition of nebulized amikacin decreases with lung aeration and severe forms of pneumonia [2,22], future RCTs should include patients at an early stage of VAP, which is often preceded by VAT. To design future RCTs, investigators should kept in mind that the European Medicines Evaluation Agency and the Food and Drug Administration approve drugs for new indications based on noninferiority and no more adverse events.

\section{CONCLUSION}

In future RCTs, high dose of nebulized amikacin (40 mg/kg/day) and nebulized colistimethate (three nebulizations of 5 million IU/day) should be delivered by nonsynchronized mesh nebulizers using volume controlled ventilation, optimized ventilator settings to limit inspiratory flow turbulences and appropriate sedation to avoid patient's asynchrony with the ventilator. Humidification and warming of inspired gas should be avoided during aerosol generation and therefore nebulization should not exceed $60 \mathrm{~min}$. Monitoring of interstitial lung concentrations in patients treated with nebulized antibiotics is difficult as ELF concentrations are not reliable due to bronchial contamination. Main objective of future RCTs performed in patients with VAP and VAT caused by XDR and pandrug resistant GNB, should be the time to obtain clinical cure rate, the duration of mechanical ventilation and the duration of antibiotic administration. Short administration of high-dose nebulized amikacin or CMS (3-5 days) should be compared with 7-day administration of new cephalosporines/betalactamase inhibitors in patients with VAP or VAT caused by XDR GNB and to intravenous CMS in patients with VAP or VAT caused by pandrug resistant GNB.

\section{Acknowledgements}

We would like to acknowledge and thank all the patients who have taken part in the trials reviewed in this article. Author contributions: J.-J.R. and A.M. wrote the first draft of the article and designed the illustrations. Y.Z., A.T., J.P. and J.R. contributed towards the critical revision of the article for important intellectual content. Each member of the European Investigators Network for Nebulized Antibiotics in Ventilator-associated Pneumonia contributed to the final article and approved its content. Appendix, http://links.lww.com/COID/A32

\section{Members of the European Research Network for Nebulized Antibiotics in Ventilator- associated Pneumonia (ENAVAP)}

Kostoula Arvaniti arvanitik@hotmail.com, Papageorgiou Hospital of Thessaloniki, Intensive Care Unit Department, Thessaloniki, Greece; Matteo Bassetti matteo.bassetti@asuiud.sanita.fvg.it, Infectious Diseases Clinic, Department of Health Sciences, University of Genoa, Genoa and Hospital Policlinico San Martino IRCCS, Genoa, Italy; Deborah Benchetrit deborah.benchetrit@aphp.fr Medicine Sorbonne University, Multidisciplinary Intensive Care Unit, Department of Anaesthesiology and Critical Care, La Pitié-Salpêtrière Hospital, Assistance Publique Hôpitaux de Paris, Paris, France; Stijn Blot stijn.blot@UGent.be, Department of Internal Medicine, Faculty of Medicine \& Health Science, Ghent University, Ghent, Belgium; Matthieu Boisson matthieu.boisson@chu-poitiers.fr, University of Poitiers, Anaesthesiology and Intensive Care Department, University hospital of Poitiers, Poitiers, France; Adrien Bouglé, adrien.bougle@aphp.fr, Medicine Sorbonne University, Anaesthesiology and Critical Care, Cardiology Institute, Department of Anaesthesiology and Critical Care, La Pitié-Salpêtrière Hospital, Assistance Publique Hôpitaux de Paris, Paris, France; Jean-Michel Constantin jean-michel.constantin@aphp.fr, Medicine Sorbonne University, Multidisciplinary Intensive Care Unit, Department of Anaesthesiology and Critical Care, La Pitié-Salpêtrière Hospital, Assistance Publique Hôpitaux de Paris, Paris, France; Jayesh Dhanani jadhanani@hotmail.com, Burns Trauma and Critical Care Research Centre and Centre for Translational Anti-infective Pharmacodynamics, The University of Queensland, Butterfield Street, Herston, Brisbane, Australia; George Dimopoulos gdimop@med.uoa.gr, Department of Critical Care Medicine, Attikon University Hospital, Medical School, National and Kapodistrian University of Athens, Athens, Greece; Jonathan Dugernier jonathan.dugernier@rhne.ch, Department of Physiotherapy, Neuchâtel hospital, Neuchâtel, Switzerland; Stephan Ehrmann stephanehrmann@gmail.com, Médecine Intensive Réanimation, CIC INSERM 1415, CRICS-TriggerSep network, CHRU Tours, Tours France and Centre d'étude des 
pathologies respiratoires INSERM U1100, Université de Tours, Tours, France; Timothy Felton timothy.felton@manchester.ac.uk, The University of Manchester and Manchester University NHS Foundation Trust, Manchester, United Kingdom; Dimitrios Karakaxas dimitrios.karakaksas@gmail.com, Department of Critical Care Medicine, Attikon University Hospital, Medical School, National and Kapodistrian University of Athens, Athens, Greece; Antonia Koutsoukou koutsoukou@yahoo.gr, Intensive Care Unit, First Department of Respiratory Medicine, School of Medicine, Sotiria General Hospital, National and Kapodistrian University of Athens, Athens, Greece; Anna Kyriakoudi Annkyr@gmail.com, Intensive Care Unit, First Department of Respiratory Medicine, School of Medicine, Sotiria General Hospital, National and Kapodistrian University of Athens, Athens, Greece; Pierre-François Laterre pierre-francois.laterre@uclouvain.be, St. Luc Clinical Coordinating Center, Department of Critical Care Medicine, St Luc University Hospital, Université Catholique de Louvain, Brussels, Belgium; Marc Leone marc.leone@ap-hm.fr, University Aix-Marseille, Department of Anaesthesiology and Critical Care, North Hospital, Marseille, France; Victoria Lepère, victoria.lepere@aphp.fr, Medicine Sorbonne University, Anaesthesiology and Critical Care, Cardiology Institute, Department of Anaesthesiology and Critical Care, La Pitié-Salpêtrière Hospital, Assistance Publique Hôpitaux de Paris, Paris, France; Gianluigi Li Bassi g.libassi@uq.edu.au Critical Care Research Group, The Prince Charles Hospital, Brisbane, University of Queensland, Faculty of Medicine, Brisbane, Australia, and Institut d'Investigacions Biomèdiques August Pi i Sunyer (IDIBAPS), Barcelona, Spain; Xuelian Liao xuelianliao@hotmail.com, Department of Critical Care Medicine, West China Hospital of Sichuan University, Chengdu, China; Olivier Mimoz o.mimoz@chu-poitiers.fr, University of Poitiers, Anaesthesiology and Intensive Care Department, University hospital of Poitiers, Poitiers, France; Lucy B Palmer, lucy.b.palmer@stonybrook.edu, Stony Brook University Medical Center Pulmonary, Critical Care and Sleep Division, SUNY at Stony Brook, HSC T17-040, Stony Brook, New York, USA; Jose Manuel Pereira, jmcrpereira@yahoo.com, Emergency and Intensive Care Department, Centro Hospitalar São João EPE, Faculdade de Medicina da Universidade do Porto, Porto, Portugal; Konstantinos Pontikis kostis_pontikis@yahoo.gr, Intensive Care Unit, First Department of Respiratory Medicine, School of Medicine, Sotiria General Hospital, National and Kapodistrian University of Athens, Athens, Greece; Garyphalia Poulakou gpoulakou@gmail.com, Third Department of Medicine, School of Medicine, Sotiria General Hospital, National and Kapodistrian University of Athens, Athens, Greece; Jieming Qu jmqu0906@163.com, Department of Pulmonary and Critical Care Medicine, Rui-jin Hospital, Shanghai Jiao-tong University School of Medicine,
Shanghai, China; Institute of Respiratory Disease, Shanghai Jiao-tong University School of Medicine, Shanghai, China; Jason Roberts j.roberts2@uq.edu.au, (1) University of Queensland Centre for Clinical Research, Faculty of Medicine \& Centre for Translational Anti-infective Pharmacodynamics, School of Pharmacy, The University of Queensland, Brisbane, Australia (2) Departments of Pharmacy and Intensive Care Medicine, Royal Brisbane and Women's Hospital, Brisbane, Australia (3) Division of Anaesthesiology Critical Care Emergency and Pain Medicine, Nimes University Hospital, University of Montpellier, Nîmes France; Christina Routsi chroutsi@hotmail.com, First Department of Intensive Care, School of Medicine, Evangelismos Hospital, National and Kapodistrian University of Athens, Athens, Greece; Candela Sole-Lleonart candela-sole@hotmail.com, Unitat Cures Intensives, Hospital Universitari de Vic, Barcelona, Spain; Melda Türkoğlu meldaturkoglu@yahoo.com.tr, Subdivision of Critical Care, Internal Medicine Intensive Care Unit, Department of Internal Medicine, Gazi University Faculty of Medicine, Ankara, Turkey; Tobias Welte welte.tobias@mh-hannover.de, University of Hannover, School of Medicine, Hannover, Germany.

\section{Financial support and sponsorship}

The current work received institutional funding from the Department of Anaesthesiology and Critical Care, La Pitié-Salpêtrière hospital, Assistance Publique Hôpitaux de Paris, Paris France.

\section{Conflicts of interest}

S.E. declares receiving consulting fees, unrestricted research grants and equipment research support from Aerogen Ltd, unrestricted research grant, speaker fees, travel reimbursements from Fisher \& Paykel, unrestricted research grant form Hamilton medical, consulting fees from La Diffusion Technique Française. M.L. is a consultant for Gilead and Amomed and gave lectures for Aspen and MSD. P.F.L. is a consultant for Adrenomed and Inotrem and receive unrestricted research grant from Aerogen. L.P. declares that Stony Brook University holds patents on targeted antibiotic therapy to intubated patients licensed to InspiRx, Inc. and that she serves as a consultant to InspiRx and is a member of Merck's Advisory Committee for Gram-negative pneumonias. $J . R$. received grant support from Bayer and served in the advisory board for Bayer and speakers bureau for Norma Helas. A.T. declares participating to the advisory board of Cardeas, Bayer and Polyphor and receiving unrestricted research grants from the three companies. $T . W$. received grant support from German Research Council, German MInistry of Research and Education, received fees for lectures from AstraZeneca, Basilea, Bayer, GSK, Infectopharm, MSD, Novartis, Pfizer, Roche and declares participating to the advisory board of 
AstraZeneca, Basilea, Bayer, GSK, Novartis, Pfizer, Roche. The other authors declare no conflict of interest.

\section{REFERENCES AND RECOMMENDED} READING

Papers of particular interest, published within the annual period of review, have been highlighted as:

- of special interest

- of outstanding interest

1. Rouby JJ, Bouhemad B, Monsel A, et al. Aerosolized antibiotics for ventilatorassociated pneumonia: lessons from experimental studies. Anesthesiology 2012; 117:1364-1380

2. Goldstein I, Wallet F, Nicolas-Robin A, et al. Lung deposition and efficiency of nebulized amikacin during Escherichia coli pneumonia in ventilated piglets. Am J Respir Crit Care Med 2002; 166:1375-1381.

3. Lu $\mathrm{Q}$, Girardi $\mathrm{C}$, Zhang $\mathrm{M}$, et al. Nebulized and intravenous colistin in experimental pneumonia caused by Pseudomonas aeruginosa. Intensive Care Med 2010; 36:1147-1155.

4. Dhanani JA, Diab S, Chaudhary J, et al. Lung pharmacokinetics of tobramycin

1. by intravenous and nebulized dosing in a mechanically ventilated healthy ovine model. Anesthesiology 2019; 131:344-355.

The experimental study in ventilated healthy ewes receiving nebulized tobramycin $400 \mathrm{mg}$, shows that epithelial lining fluid (ELF) concentrations grossly overestimate interstitial space fluid concentrations measured by microdialysis.

5. Kollef $\mathrm{MH}$, Ricard JD, Roux D, et al. A randomized trial of the amikacin fosfomycin inhalation system for the adjunctive therapy of Gram negative ventilator-associated pneumonia: IASIS trial. Chest 2017; 151:1239- 1246.

6. Niederman MS, Alder J, Bassetti $M$, et al. Inhaled amikacin adjunctive to

- intravenous standard-of-care antibiotics in mechanically ventilated patients with Gram-negative - pneumonia (INHALE): a double blind, randomised, placebo-controlled, phase 3, - superiority trial. Lancet Infect Dis 2020; 20:330-340.

The INHALE multicentre double-blind randomized controlled study shows that the nebulization of amikacin $400 \mathrm{mg}$ twice a day during 10 days using an inspirationsynchronized mesh nebulizer and combined with one or two intravenous antibiotics, is not superior to standard intravenous antibiotherapy to treat ventilator associated pneumonia caused by sensitive ( $50 \%$ of patients) or multidrug resistant (50\% of patients) Gram-negative bacteria (GNB)

7. Stokker J, Karami M, Hoek R, et al. Effect of adjunctive tobramycin inhalation versus placebo on early clinical response in the treatment of ventilatorassociated pneumonia: the VAPORISE randomized controlled trial. Int Care Med 2020; 46:546-548.

8. Kalil AC, Metersky ML, Klompas M, et al. Executive summary: management of adults with hospital-acquired and ventilator associated pneumonia: 2016 clinical practice guidelines by the Infectious Diseases Society of America and the American Thoracic Society. Clin Infect Dis 2016; 63:575-582.

9. Rello J, Solé-Lleonart C, Rouby JJ, et al. Use of nebulized antimicrobials for the treatment of respiratory infections in invasively mechanically ventilated adults: a position paper from the European Society of Clinical Microbiology and Infectious Diseases. Clin Microbiol Infect 2017; 23:629-639.

10. Torres A, Niederman MS, Chastre J, et al. International ERS/ESICM/ESCMID/ ALAT guidelines for the management of hospital-acquired pneumonia and ventilator-associated pneumonia. Eur Respir J 2017; 50:1700582.

11. Leone $\mathrm{M}$, Bouadma $\mathrm{L}$, Bouhemad $\mathrm{B}$, et al. Hospital-acquired pneumonia in ICU. Anaesth Crit Care Pain Med 2018; 37:83-98.

12. Sweeney DA, Kalil AC. Why don't we have more inhaled antibiotics to treat ventilator-associated pneumonia? Clin Microbiol Infect 2019; 25:1195-1199.

13. Rouby JJ, Sole-Lleonart C, Rello J; European Investigators Network for

- Nebulized-Antibiotics in Ventilator-associated Pneumonia. Ventilator-associated pneumonia - caused by multidrug-resistant Gram-negative bacteria: understanding nebulization of - aminoglycosides and colistin. Intensive Care Med 2020; 46:766-770.

The review on behalf of the members of the European Investigators Network for Nebulized Antibiotics for Ventilator-Associated Pneumonia shows that substitution therapy should be preferred to adjunctive therapy to show the benefit of nebulized aminoglycosides and colistin in ventilator-associated pneumonia (VAP) caused by multidrug resistant GNB and that mesh nebulizers should be preferred to jet nebulizers.

14. Rodvold KA, George JM, Yoo L. Penetration of antiinfective agents into pulmonary epithelial lining fluid: focus on antibacterial agents. Clin Pharmacokinet 2011; 50:637-664.

15. Morais CLM, Nascimento JWL, Ribeiro AC, et al. Nebulization of vancomycin

- provides - higher lung tissue concentrations than intravenous administration in ventilated - female piglets with healthy lungs. Anesthesiology 2020; 132:1516-1527.

The experimental study performed in ventilated healthy female animals shows that the nebulization of vancomycin $37.5 \mathrm{mg} / \mathrm{kg}$ results in vancomycin lung tissue concentrations 13 -fold higher than those resulting from the intravenous administration of $15 \mathrm{mg} / \mathrm{kg}$.
16. Paul M, Lador A, Grozinsky-Glasberg S, Leibovici L. Beta lactam antibiotic mono therapy versus beta lactam-aminoglycoside antibiotic combination therapy for sepsis. Cochrane Database Syst Rev 2014; 2014 CD003344.

17. Arthur LE, Kizor RS, Selim AG, et al. Antibiotics for ventilator-associated pneumonia. Cochrane Database Syst Rev 2016; 10:CD004267.

18. Abad CL, Kumar A, Safdar N. Antimicrobial therapy of sepsis and septic shock-when are two drugs better than one? Crit Care Clin 2011; 27:e1-e27.

19. Magiorakos AP, Srinivasan A, Carey RB, et al. Multidrug-resistant, extensively drug-resistant and pandrug-resistant bacteria: an international expert proposal for interim standard definitions for acquired resistance. Clin Microbiol Infect 2012; 18:268-281.

20. Palmer LB, Smaldone GC. Reduction of bacterial resistance with inhaled antibiotics in the intensive care unit. Am J Respir Crit Care Med 2014 189:1225-1233.

21. Li Bassi G, Motos A, Fernandez-Barat $L$, et al. Nebulized amikacin and fosfomycin for severe Pseudomonas aeruginosa pneumonia: an experimental study. Crit Care Med 2019; 47:e470-e477.

22. Elman $M$, Goldstein I, Marquette $\mathrm{CH}$, et al. Influence of lung aeration on pulmonary concentrations of nebulized and intravenous amikacin in ventilated piglets with severe bronchopneumonia. Anesthesiology 2002; 97: 199-206.

23. Goldstein I, Bughalo MT, Marquette $\mathrm{CH}$, et al., Experimental ICU Study Group. Mechanical ventilation-induced air-space enlargement during experimental pneumonia in piglets. Am J Respir Crit Care Med 2001; 163: 958-964.

24. Dugernier J, Ehrmann S, Sottiaux T, et al. Aerosol delivery during invasive mechanical ventilation: a systematic review. Crit Care 2017; 21:264.

25. Ari A, Dang T, Al Enazi FH, et al. Effect of heat moisture exchanger on aeroso drug delivery and airway resistance in simulated ventilator-dependent adults using jet and mesh nebulizers. J Aerosol Med Pulm Drug Deliv 2018; $31: 42-48$.

26. Miller DD, Amin MM, Palmer LB, et al. Aerosol delivery and modern mechanical ventilation: in vitro/in vivo evaluation. Am J Respir Crit Care Med 2003 168:1205-1209.

27. Rouby JJ, Monsel A. Nebulized antibiotics: epithelial lining fluid concentrations

- overestimate lung tissue concentrations. Anesthesiology 2019; 131: $229-232$.

The editorial describes the data suggesting that ELF concentrations of nebulized antibiotics grossly overestimate interstitial lung fluid concentrations due to massive bronchial contamination of the fiberscope during the bronchoalveolar lavage procedure.

28. Marchand S, Gregoire N, Brillault J, et al. Biopharmaceutical characterization of nebulized antimicrobial agents in rats: 3 . Tobramycin. Antimicrob Agents Chemother 2015; 59:6646-6647.

29. Landersdorfer CB, Nguyen $T H$, Lieu LT, et al. Substantial targeting advantage achieved by pulmonary administration of colistin methanesulfonate in a large-animal model. Antimicrob Agents Chemother 2016; 61:e01934e1941.

30. Boisson $\mathrm{M}$, Jacobs $\mathrm{M}$, Gregoire $\mathrm{N}$, et al. Comparison of intrapulmonary and systemic pharmacokinetics of colistin methanesulfonate (CMS) and colistin after aerosol delivery and intravenous administration of CMS in critically ill patients. Antimicrob Agents Chemother 2014; 58:73317339

31. Luyt $C E$, Clavel $M$, Guntupalli $K$, et al. Pharmacokinetics and lung delivery of PDDS aerosolized amikacin (NKTR-061) in intubated and mechanically ventilated patients with nosocomial pneumonia. Crit Care 2009; 13:R200.

32. Kelly CA, Kotre CJ, Ward C, et al. Anatomical distribution of bronchoalveola lavage fluid as assessed by digital subtraction radiography. Thorax 1987; 42:624-628.

33. Rodvold KA, Hope WW, Boyd SE. Considerations for effect site pharmacokinetics to estimate drug exposure: concentrations of antibiotics in the lung Curr Opin Pharmacol 2017; 36:114-123.

34. Athanassa ZE, Markantonis SL, Fousteri MZ, et al. Pharmacokinetics of inhaled colistimethate sodium (CMS) in mechanically ventilated critically ill patients. Intensive Care Med 2012; 38:1779-1786.

35. Solé-Lleonart $\mathrm{C}$, Rouby JJ, Blot $\mathrm{S}$, et al. Nebulization of antiinfective agents in invasively mechanically ventilated adults: a systematic review and metaanalysis. Anesthesiology 2017; 126:890-908.

36. Rello J, Rouby JJ, Sole-Lleonart C, et al. Key considerations on nebulization of antimicrobial agents to mechanically ventilated patients. Clin Microbiol Infect 2017; 23:640-646.

37. Dhand R, Sohal H. Pulmonary drug delivery system for inhalation therapy in mechanically ventilated patients. Expert Rev Med Devices 2008; 5:9-18

38. Rouby JJ, Monsel A, Ehrmann S, et al. The INHALE trial: multiple reasons for a negative result. Lancet Infect Dis 2020; 20:778-779.

The letter describes the mechanisms by which inspiration-synchronized mesh nebulizers are unable to deliver appropriate dose of amikacin to patients with VAP.

39. Lu Q, Yang J, Liu Z, et al. Nebulized ceftazidime and amikacin in ventilatorassociated pneumonia caused by Pseudomonas aeruginosa. Am J Resp Crit Care Med 2011; 184:106-115. 


\section{Respiratory infections}

40. Dugernier J, Reychler G, Wittebole X, et al. Aerosol delivery with two ventilation modes during mechanical ventilation: a randomized study. Ann Intensive Care 2016; 6:73.

41. Mashat $M$, Clark BJ, Assi $\mathrm{KH}$, Chrystyn $\mathrm{H}$. In vitro aerodynamic characterization of the dose emitted during nebulization of tobramycin high strength solution by novel and jet nebulizer delivery systems. Pulm Pharmacol Ther 2016; 37:37e42.

42. Chang $\mathrm{KH}$, Moon $\mathrm{SH}$, Oh JY, et al. Comparison of salbutamol delivery effciency for jet versus mesh nebulizer using mice. Pharmaceutics 2019; 11:192.

43. Liu CY, Ko HK, Fink JB, et al. Size distribution of colistin delivery by different type nebulizers and concentrations during mechanical ventilation. Pharmaceutics 2019; 11:459.

44. Galindo-Filho VC, Alcoforado L, Rattes $C$, et al. A mesh nebulizer is more effective than jet nebulizer to nebulize bronchodilators during noninvasive ventilation of subjects with COPD: a randomized controlled trial with radiolabeled aerosols. Respir Med 2019; 153:60-67.
45. Dunne RB, Shortt S. Comparison of bronchodilator administration with vibrating mesh nebulizer and standard jet nebulizer in the emergency department. Am J Emerg Med 2018; 36:641-646.

46. Gowda AA, Cuccia D, Smaldone GC. Reliability of vibrating mesh technology Respir Care 2016; 62:65-69.

47. Skaria S, Smaldone GC. Omron NE U22: comparison between vibrating mesh and jet nebulizer. J Aerosol Med Pulm Drug Deliv 2010; 23 . $173-180$.

48. Torres A, Motos A, Battaglini D, Li Bassi G. Inhaled amikacin for severe Gram negative pulmonary infections in the intensive care unit: current status and future prospects. Crit Care 2018; 22:343.

49. Palmer LB. Why have trials of inhaled antibiotics for ventilator-associated infections failed? Curr Opin Infect Dis 2019; 32:163-168.

50. Rouby JJ, Monsel A, Leone M, et al. The IASIS, INHALE and VAPORISE trials. Reasons for a triple failure: study design, aminoglycosides dosing and technique of nebulisation. Anaesth Crit Care Pain Med 2020; 39:179-183. 